\title{
The machine as intermediary: personal communication via a machine ${ }^{\dagger}$
}

\author{
Digby Tantam
}

\begin{abstract}
The physical co-presence of psychotherapist or counsellor and client has been considered essential to the development of the therapeutic relationship. Psychotherapists have often, therefore, been resolutely opposed to anything other than face-to-face therapy. The richness and ubiquity of internet communication is likely to change that. I consider here the circumstances in which internet psychotherapy is indicated, some of the new problems it poses, including boundary-keeping and ethics, along with some solutions to these problems. The potential of the internet has already been exploited by many clients themselves and I conclude with a consideration of the growth of internet-based peer support groups.
\end{abstract}

This article concludes Digby Tantam's discussion in APT of electronic media in psychotherapy (see also Tantam, 2006a,b). The third article in this series of four, in which he considers communication with, rather than via, the machine also appears in this issue. ${ }^{+}$

All of the new technologies discussed in my first article in this series (Tantam, 2006a) have been used by some 'early adopters', who have embraced one or other of them for support, psychotherapy, supervision or training (Castelnuovo et al, 2003). Psychotherapists have used these media to maintain contact with their clients as an adjunct to face-to-face psychotherapy (Murdoch \& Connor-Greene, 2000) or as a means of continuing therapy while either the client or the therapist is away from their base. People with problems have used electronic media to find the psychotherapist that they want, irrespective of their distance, to fit round an otherwise inflexible schedule or to overcome geographical isolation (Tantam et al, 2002). Supervisees in isolated locations similarly benefit from e-supervision (Wood et al, 2005). People with problems have also been able to find fellow sufferers, and the internet in particular has provided the opportunity for highly specialised online support networks to develop. These have been used as an alternative to therapy by individuals who would not consider themselves sick enough

'See pp. 416-426, this issue. to seek a psychotherapist, but also by people who have more problems, or are more disturbed, than the clients that many psychotherapists would consider suitable (Leiberich et al, 2004).

\section{E-therapy}

At the moment, e-therapy is not widespread. One relatively recent study concluded that the number of psychologists offering e-therapy is small, their services are diverse, and their compliance with established ethical principles is uneven' (Heinlen et al, 2003). However, the situation is changing rapidly. The American Medical Association added a Current Procedural Terminology code for online medical evaluation in July 2004, and from that date reimbursement has been possible in the USA for all online medical consultations, including counselling and psychotherapy. There is also an increasing number of virtual offices in which psychotherapists can rent space (e.g. http:/ / www.etherapistsonline. com and http: / / www.helphorizons.com in the USA and http: / / www.etherapy.com.au in Australia) and introductory sites that put potential clients in touch with therapists. The latter are usually commercial sites (e.g. http://www.find-a-therapist.com), but one of the earliest was developed as a service to the gay and lesbian community in London (http:/ / www.pinkpractice.co.uk).

Digby Tantam is Clinical Professor of Psychotherapy at the University of Sheffield (Centre for the Study of Conflict and Reconciliation, University of Sheffield School of Health and Related Research, 30 Regent Street, Sheffield S6 6GJ, UK. Email: d.tantam@sheffield.ac.uk). $\mathrm{He}$ is Co-Director of the University's Centre for the Study of Conflict and Reconciliation, Director of the Section of Mental Health within the School of Health and Related Research, and Deputy Director of Teaching for the School. He is also an honorary consultant psychotherapist and psychiatrist in Sheffield Care Trust. His current research interests include the evaluation of internet-based learning and teaching. 
It has been claimed (Ainsworth, 2002) that the first use of e-therapy was Ask Uncle Ezra, developed in 1986 at Cornell University (http:/ / ezra.cornell.edu/ ask.php) and acting as a helpline ever since. This is an asynchronous discussion forum providing advice rather than counselling. Founders of well-known sites such as Ivan Goldberg, who set up Depression Central (http://www.psycom.net/depression.central. html), and John Grohol, who founded Psych Central (http://psychcentral.com), have provided free mental health advice and support, but e-counselling and e-therapy seem to have developed only in the mid-1990s. The Samaritans extended their telephone counselling to email in 1994 (email: jo@samaritans. org), and several individual therapists began to use secure internet chat as a medium for therapy, although on a fee-for-service basis, from about the same period.

Some obvious practical problems of e-therapy are considered in Box 1. As regards security and confidentiality of communication, hacking is probably no more serious than having someone eavesdrop at your office door, and clients can find security procedures off-putting (Tjora et al, 2005). A problem to which there is no easy solution is that of clients who drop out of therapy (see section on Ethics below). This seems particularly common in e-therapy, perhaps because of the anonymity of the process or because the therapist lacks the particular skill necessary to make e-therapy work (Carlbring et al, 2003).

\section{Box 1 Dealing with some practical problems} of e-therapy and e-counselling

- Fees: bill per item of asynchronous service and bill per hour for synchronous services

- Records: keep a record of all electronic communications

- Security and confidentiality: store files either encrypted or in an offline medium such as CD in a filing cabinet, make sure that your server's security is kept up to the most modern levels

- Insurance: check that your indemnity covers internet work

- Dealing with attempted fraud or impersonation: get clients to email to you their unique name and password (you can then check their email or contact address); if possible, arrange payment by credit card so that the client's identity can be confirmed by the credit card company; have a contract with the client setting out boundary issues
These issues have been discussed in a number of books (e.g. Kraus et al, 2004) and in the excellent guidelines of the British Association of Counselling and Psychotherapy (Goss et al, 2002). There are also some more principled problems, about ethics, about anonymity and about effectiveness, that affect both e-therapy and e-supervision. They arise from what have become familiar themes in this series of articles - disembodiment and the asynchronicity of some internet interactions. The new electronic media pose the same challenges in all areas (e-learning, etherapy, e-supervision and so on), but for simplicity I concentrate here on how they affect internet-based counselling and psychotherapy.

\section{Asynchronous communication}

Asynchronous communication has the advantage that the client can post a message exactly when they feel the pinch, when their problem or difficulty is clearest to them. Having written it, they may go back and edit or amend it (if it is posted on a discussion forum, but not if sent as an email) before it is read by the therapist, enabling second or even third thoughts.

\section{Boundaries}

A face-to-face meeting provides both the therapist and the client with considerable information about boundaries. They know when other people are present and can judge whether anyone else is listening. They can gauge whether or not the other is intoxicated, unwell, drowsy, attentive. The therapist in particular will be aware of the passage of time and the intended duration of the session. Virtual interaction has only those boundaries that are contracted between the participants. It may be impossible to know if it is always the same person who is participating in the therapy, if there are witnesses to the therapeutic exchange, or if the client is engaged and attentive, or occupied with some other task, intoxicated or otherwise distracted. Boundary violations are therefore a particular problem.

The main way of getting round this is to spend more time developing the therapeutic relationship and establishing the basis of trust. Negotiating a contract with the client, giving details about what is expected of both parties, before therapy begins is one important way of dealing with this. Selection is probably also more important in e-therapy and e-supervision (Suler, 2001) and there may be an argument for meeting the potential client or supervisee or, at the very least, having a telephone conversation with them. 


\section{Ethics}

Ethical and legal problems associated with e-therapy have concerned many professionals (Manhal-Baugus, 2001), and clear-cut guidelines have been developed, for example by the International Society for Mental Health Online (2000) and by an increasing number of counselling organisations such as the British Association of Counselling and Psychotherapy (Goss et al, 2002). Many of these address the risks of impersonation or fraud, but how much of a risk these present is not yet clear. It does seem essential, however, that therapists agree a security protocol with clients, similar to that operated by many online shopping sites, which does as much as possible to ensure that the person who is using the computer at the other end is actually the client.

There may be fewer unique ethical dilemmas in e-therapy and e-supervision than appear. Issues that are often raised, for example efficacy, the risk of side-effects such as positive transference (Gabbard, 2001), informed consent, information about 'standard operating procedure', what to do in an emergency (Hsiung, 2002) and the dangers of misunderstanding the client's communications, all arise in face-to-face psychotherapy. However, a survey of e-therapy sites conducted a few years ago (Heinlen et al, 2003) concluded that many fell short of standards such as those of the International Society for Mental Health Online (2000) and the American Psychological Association (http://www.apa.org).

Many writers about e-therapy emphasise the importance of training and the potential challenges of e-therapy to inexperienced practitioners. It is my experience that technique does have to be modified, but no more so than working with a client with a sensory impairment or across a cultural divide. Since these obstacles are assumed to be ones that all therapists should expect to be able to negotiate, e-therapy, too, should be well within the range of the average psychotherapist's skills.

Perhaps the most challenging ethical requirement is to ensure that the client-therapist relationship is maintained for an adequate time. E-therapy has a higher level of drop out than face-to-face therapy (Carlbring et al, 2003). Not only can this make it difficult for the therapist to discharge the duty of care that their relationship with the client imposes, whether or not it is mediated electronically, but it can be disturbing for the therapist to 'lose' a client unexpectedly and completely. It is even more disturbing for the client to lose their therapist unexpectedly and it is therefore alarming that Heinlen et al (2003) found that internet therapy services are often unexpectedly discontinued by the service provider.
Box 2 Preparing for and preventing ethical problems

The clinician should:

- undertake appropriate training and professional preparation

- decide which sources of referrals to accept

- provide information to educate potential clients about the therapy

- have an adequate procedure for obtaining consent

- assess the client (and be assessed by them)

- determine the appropriate method of etherapy and the mix of e-therapy and personal therapy

- determine an appropriate and agreed method of reimbursement

Maheu (2003)

The lack of presence that I have already discussed in relation to training (this issue, pp. 416-426) applies equally to therapy. It is easy to overlook a lack of involvement of clients or supervisees when they are not present in the room, especially when therapy or supervision is being conducted in a group. The therapist must take particular care to ensure that this does not happen. Both client and therapist need to be willing to work to overcome this, and this may make e-therapy as the sole treatment modality suitable only for a minority of clients. It may well be that blended therapy, incorporating both electronic and face-to-face contact, is a better solution than either one or the other.

Box 2 outlines a useful framework for preparing for and preventing possible ethical problems, proposed by Maheu (2003). Her article concludes with a useful informed consent agreement for telehealth.

\section{Online support groups}

Psychotherapy is an emotionally demanding procedure for both client and therapist. It requires disclosure, and this inevitably involves the risk of shame or humiliation that can never, despite every proof and protestation of the therapist, be entirely assuaged. There is, too, the feeling that therapists cannot really know a client's pain. They seem too ensconced in their own comfortable and emotionally secure world. Therapists are, perhaps, less comfortable than they seem, not least because theirs is a slender thread by which to help clients through the squalls of life. Therapists who work in a private practice or in a specialist psychotherapy 
service in the NHS rarely have the kind of contract with their clients that would legitimise arranging a home visit, asking a community nurse to make a follow-up visit or asking the client to make contact with the crisis intervention team. Therapists are therefore often careful, arguably sometimes too careful (Tantam, 1995), to select clients who are 'suitable' for therapy. Managed care in the USA and the National Service Framework for Mental Health in the UK (Department of Health, 1999) have also meant that mental health services have been focused on only some groups of people with mental health problems, primarily those for whom medical treatments are well-established.

All of these different factors mean that there are substantial numbers of people who have significant mental health problems but who cannot access services. There may be many reasons for this. The excluded group will include people who may not have been willing or able to engage in the past. Also excluded will be people who have been violent, self-destructive or troublesome in some other way; people whose conditions are deemed refractory to treatment or are outside the treatment priorities; and people who feel exposed or violated by the treatment process itself. Many will have conditions that are stigmatised.

Mental health charities have been the main alternative to conventional treatment, but the growth of the internet is providing another source of support. Websites have been created by people with particular disorders for the benefit of others with the disorder (for a directory see http://www.psychcentral. com/resources). Many of them incorporate discussion forums (e.g. http://p199.ezboard. $\mathrm{com} /$ bcopingwithdissociativeidentitydisorder for people with dissociative disorders, and the Germanlanguage site for people with eating disorders, http://www.hungrig-online.de). However, it is usenet groups (Tantam, 2006a) that have provided the main source of specialist support networks. Usenet groups are archived on the World Wide Web and can be accessed by a browser, but most exist outside it. Access to them is via a subscription, which is determined by the user's internet service provider. This is the seedy side of the net. Many usenet groups distribute pornography or 'cracks', i.e. passwords to or illegal copies of games or software. But this is where support groups are also to be found. Many are classified under 'alt.support'. Usenet groups may require registration, and some, but not all, may be moderated.

Anecdotal evidence suggests that peer support can be both helpful and unhelpful. There is no systematic evidence (Eysenbach et al, 2004) and it will be important in the future for professionals to become more involved in the provision of information and support, and in their evaluation, to ensure that benefits outweigh harm (Eysenbach et al, 2001).

\section{Conclusions}

Electronic psychotherapy is probably here to stay. Its limitations and its advantages are becoming clearer, as I have summarised in this series of articles. Its clearest application is as an extension, adjunct or supplement to psychotherapy.

The internet has already been put to use by clients, or potential clients, to exchange information, help and advice, including advice about psychotherapy services. The Royal College of Psychiatrists (http:/ / www.rcpsych.ac.uk) is beginning to make its own contribution to this rich stew of information, but there is considerable potential for individual psychotherapists, practices, clinics and organisations to do a great deal more. This is likely to become an important marketing tool in the future, but it will also be a medium by which clinicians can disseminate information about good practice and about good mental health.

Finally, the impact of e-learning on training is only just beginning to be seen. The collapse of the UK government's e-learning initiative has led some to believe that e-learning itself is a false start. My own experience in the field suggests rather that its rise may be slow, even insidious, but that it will also be irresistible. A new Gutenbergian revolution is in progress.

\section{Declaration of interest}

D.T. is the author of a hypermedia distance-learning course SEPTIMUS (http:/ / www.septimus.info). He was also a director of InterPsych, the network of discussion forums on mental health created by Ian Pitchford (http:/ / human-nature.com/pitchford).

\section{References}

Ainsworth, M. (2002) My life as an e-patient. In E-Therapy: Case Studies, Guiding Principles and the Clinical Potential of the Internet (ed. R. Hsiung), pp. 194-216. New York: W. W. Norton.

Carlbring, P., Ekselius, L. \& Andersson, G. (2003) Treatment of panic disorder via the Internet: a randomized trial of CBT vs. applied relaxation. Journal of Behavior Therapy and Experimental Psychiatry, 34, 129-140.

Castelnuovo, G., Gaggioli, A., Mantovani, F., et al (2003) From psychotherapy to e-therapy: the integration of traditional techniques and new communication tools in clinical settings. CyberPsychology and Behavior, 6, 375-382.

Department of Health (1999) National Service Framework for Mental Health: Modern Standards and Service Models. London: Department of Health.

Eysenbach, G., Köhler, C., Yihune, G., et al (2001) A framework for improving the quality of health information on the worldwide-web and bettering public e-health. The MedCERTAIN 
approach. In Medinfo01, Proceedings of the Tenth World Congress on Medical Informatics (eds R. Haux, V. Patel \& A. Hasmann), pp. 1450-1454. Elsevier.

Eysenbach, G., Powell, J., Englesakis, M., et al (2004) Health related virtual communities and electronic support groups: systematic review of the effects of online peer to peer interactions. BMJ, 328, 1166.

Gabbard, G. O. (2001) Cyberpassion: E-rotic transference on the Internet. Psychoanalytic Quarterly, 70, 719-737.

Goss, S., Palmer, S., Jamieson, A., et al (2002) Online Counselling Guidelines. Rugby: British Association of Counselling and Psychotherapy.

Heinlen, K., Welfel, E., Richmond, E., et al (2003) The nature, scope, and ethics of psychologists' e-therapy web sites: what consumers find when surfing the web. Psychotherapy Theory, Research, Practice, Training, 40, 112-124.

Hsiung, R. (2002) E-therapy: Case Studies, Guiding Principles and the Clinical Potential of the Internet. New York: W. W. Norton.

International Society for Mental Health Online (2000) Suggested Principles for the Online Provision of Mental Health Services. Version 3.11. Foster City, CA: ISMHO. http://www.ismho. org/suggestions.html

Kraus, R., Zack, J. \& Stricker, G. (eds) (2004) Online Counseling: A Handbook for Mental Health Professionals. San Diego, CA: Elsevier.

Leiberich, P., Nedoschill, J., Nickel, K., et al (2004) Internet communication: new kind of self-help improves access to psychotherapy for eating disorders. Journal of Psychosomatic Research, 56, 614-615.

Maheu, M. M. (2003) The online clinical practice management model. Psychotherapy Theory, Research, Practice, Training, 40, 20-32.

Manhal-Baugus, M. (2001) E-therapy: practical, ethical, and legal issues. CyberPsychology and Behavior, 4, 551-563.

Murdoch, J. W. \& Connor-Greene, P. A. (2000) Enhancing therapeutic impact and therapeutic alliance through electronic mail homework assignments. Journal of Psychotherapy Practice and Research, 9, 232-237.

Suler, J. (2001) Assessing a person's suitability for online therapy: the ISMHO clinical case study group. CyberPsychology and Behavior, 4, 675-679.

Tantam, D. (1995) Why select? In The Art and Science of Psychotherapy Assessment (ed. C. Mace), pp. 1-30. London: Routledge.

Tantam, D. (2006a) Computers, the internet and the World Wide Web: an introduction for the e-therapist. Advances in Psychiatric Treatment, 12, 359-367.

Tantam, D. (2006b) Opportunities and risks in e-therapist. Advances in Psychiatric Treatment, 12, 368-374.

Tantam, D., van Deurzen, E. \& Osterloh, K. (2002) The Survey of European Psychotherapy Training. 2: Questionnaire data. European Journal of Psychotherapy, Counselling and Health, 4, 379-396.

Tjora, A., Tran, T. \& Faxvaag, A. (2005) Privacy vs usability: a qualitative exploration of patients' experiences with secure internet communication with their general practitioner. Journal of Medical Internet Research, 7, e15.

Wood, J. A. V., Miller, T. W. \& Hargrove, D. S. (2005) Clinical supervision in rural settings: a telehealth model. Professional Psychology: Research and Practice, 36, 173-179.

\section{MCQs}

1 Computers:

a are likely to replace all psychotherapists in the future

$\mathrm{b}$ are likely to be used as adjunctive communication devices by all psychotherapists in the future

c provide insuperable problems of security

$\mathrm{d}$ are not going to be used by the most disturbed clients.

2 It may be impossible to establish definitively the identity of a client met only over the internet. This:

a creates unique problems never encountered in faceto-face therapy

b means that e-therapy will be efficacious only if combined with face-to-face therapy

c requires modifications to administrative procedures

$\mathrm{d}$ will make many psychotherapists chary of etherapy.

\section{Peer support groups:}

a can only be of benefit

$b$ may be the only means of accessing help for some people

c are rarely hosted on internet sites outside the World Wide Web

d exist for many different health conditions.

\section{MCQ answers}

\begin{tabular}{|c|c|c|}
\hline 1 & 2 & 3 \\
\hline a F & a F & a $F$ \\
\hline $\mathrm{b} \mathrm{T}$ & b F & b $\mathrm{T}$ \\
\hline c F & c $\mathrm{T}$ & c F \\
\hline d F & d $\mathrm{T}$ & d F \\
\hline
\end{tabular}

